\title{
Evaluation of Fine Needle Aspiration Cytology of Cervical Lymphadenopathies at Tertiary Care Center
}

\author{
Pravin Gojiya, Alpeshpuri Goswami*, and Shaila Shah
}

Department of Pathology, Government Medical College \& Sir Takhtasinhji Hospital, Bhavnagar (India)

\begin{abstract}
Background: Lymphadenopathies in the neck region is the most frequently sent for the cytology evaluation from the clinicians, involvement in regional and systemic diseases and their easy accessibility make the FNAC as a primary workup. Inflammatory and immune reactions are the most frequent causes of lymph node enlargement and are self limiting in majority of cases. Tuberculosis can also be diagnosed by cytology of affected lymph nodes. With the advent of FNAC, most of the inflammatory, reactive and neoplastic conditions can be diagnosed without biopsy. It has the advantage that it can be done safely, rapidly and cheaply with minimal trauma at an outpatient setup or at the bedside.

Methods: This study was conducted at cytology section of pathology department of our institute. Patients from ENT, Surgery, Medicine, Pulmonary medicine departments were referred for FNAC. Written informed consent was obtained from all patients. It included patients with lymphadenopathies in cervical region. FNAC was conducted with the help of a 24 guage disposable needle attached to a $10 \mathrm{cc}$ syringe. Smears were fixed in methanol and stained with Haematoxylin and Eosin as well as Papanicolaou stains. MGG stain was done on air dried smears. The results expressed as percentage were tabulated.

Result: Maximum numbers of patients were diagnosed with Reactive Lymphadenopathy 67/266 (25\%), followed by Chronic Granulomatous Inflammation 59/266 (22\%), Metastatic 52/266(20\%), Acute Suppurative 37/266 (14\%), Tuberculous 27/266 (10\%), Non Specific 14/266 (5 \%), Lymphoma (primary) 8/266 (3\%) and miscellaneous 2/266 (1\%) consists Sinus Histiocytosis \& Kawashaki disease. Histopathological correlation was available in $25 / 266$ cases.
\end{abstract}

Conclusion: FNAC is a safe, simple \& inexpensive primary immediate diagnostic procedure and workup for lymph node enlargements, especially in cervical lymphadenopathies where biopsies are not done routinely.

\section{Keywords: Cytology, Cervical Lymph Nodes, Metastatic Malignancies}

\section{Introduction}

Cervical lymphadenopathy is commonest clinical presentations of patients, attending the outdoor clinics in most hospitals. The etiology varies from an inflammatory cause to a malignant condition ${ }^{[1,3]}$. Fine needle aspiration cytology (FNAC) of lymph node has become the initial workup in diagnosis and management of patients with lymphadenopathy due to early availability of results, simplicity and minimal trauma with less complication ${ }^{[4]}$. FNAC has also been advocated as a useful method in comparison to more expensive surgical excision biopsies in developing countries with limited financial and health care resources ${ }^{[5]}$. It almost offers an accurate diagnosis for infectious disease, granulomatous lymphadenitis, reactive lymphoid hyperplasia and metastatic malignancy. Thus, it can avoid the need for excision biopsy in most cases and allow rapid onset of therapy ${ }^{[6]}$. The diagnosis of metastatic tumor to the lymph node on cytological smear is crucial and highly reliable. This would be the sole indication for searching the primary tumor, especially in cases of occult carcinoma ${ }^{[7]}$. However, in most of these cases, the primary tumor is clinically known and FNAC is used widely for the follow up of these patients. Most of metastatic carcinoma can be identified by their cytomorphological characteristics alone. However, there are some instances where features of different tumors overlap and the precise diagnosis of the primary tumor remains obscure ${ }^{[8]}$. Ancillary techniques, such as immunocytochemistry, are used to overcome these difficulties and support the cytodiagnostic interpretation ${ }^{[9]}$.

FNAC is used mainly to assess the staging of primary lymphoid malignancies as well as to recognize the residual and recurrent lymphoid malignancies ${ }^{[10]}$. Shakya et al. ${ }^{[11]}$ also mentioned that the cytology is more readily accepted for the evaluation of deeply seated lymph nodes (i.e. surgically inaccessible) with primary lymphoma or for medically unfit patients for surgery. However, the role of FNAC for the initial diagnosis and subclassification of primary lymphoid malignancy is still controversial 
and the cytological diagnosis of lymphoma on FNAC is still very often followed by tissue biopsy in most cases ${ }^{[12]}$. Since the latest World Health Organization (WHO) lymphoma classification is based not only on the architectural pattern, but also on cellular morphology, phenotype, and genotype of malignant lymphoid cells; and all of which can be assessed on cytology. Therefore, FNAC in combination with immunophenotypic and genotypic studies is gaining respect in providing an accurate diagnosis of malignant lymphoma in selected risk patients ${ }^{[13]}$. Cervical lymph nodes are involved most often in all types of lymphadenopathy particularly reactive hyperplasia and Hodgkin lymphoma [11]. Although the reliability of FNAC of cervical lymph node has been shown in some studies ${ }^{[14,15]}$ but there are also some reports in contrary ${ }^{[16,17]}$.

\section{Materials and Methods}

This retrospective study on 266 selected patients with cervical lymphadenopathy was conducted at the Cytology section, Pathology Department of our institute from January 2015 to December 2015. Cervical nodal enlargement was the clinical manifestation of the patients in all cases. In this study is of FNAC from the enlarged cervical lymph nodes done, which were stained with Hematoxylin and Eosin as well as Papanicolaou stains. MGG stain was done on air dried smears. In each studied case, a brief clinical history was considered including age, sex, size and site of enlarged cervical nodes. The cytology smears of all cases were examined to determine the cytomorphological features. These features included material adequacy, cellular populations, cell arrangement and nuclear as well as cytoplasmic features. The background was noted for the presence of necrosis and grannuloma formation as well as for the type of inflammatory cells.

Paucicellular smears were excluded from the present study. The cytology results included four main diagnoses; (a) "benign diagnosis with recommendation of follow up" that deal with smears presenting no malignant tumor cells. (b) "Malignant metastatic diagnosis with recommendation of searching for the primary tumors" that comprised smears showing malignant metastatic tumor cells. (c) "Malignant primary lymphoma (non Hodgkin lymphoma, large cell type or Hodgkin lymphoma) with recommendation of excision for confirmation and immunophenotyping" that showed large malignantlooking lymphoid cells or typical Reed-Sternberg (R-S) cells. (d) "Suggestive of or suspicious for lymphoid malignancy with a recommendation of biopsy and immunophenotyping" that revealed atypical small or large lymphoid cells or revealed R-S and Hodgkin-like cells. In the current study, we considered suggestive or suspicious cases as positive for malignancy as all these cases were investigated and managed seriously.

The histopathological assessment was advocated in the included cytologically benign cases either due to clinical persistent, multiple, or enlarging lymphadenopathy or due to suspicious radiological or laboratory features. The histopathological examination was performed in the included malignant metastatic cases as the metastatic work up of such cases failed to identify the primary tumors. The cytopathological diagnoses then were compared with the histopathological results of the same excised nodes. In cases of discrepancy, histopathologic results were considered the gold standard.

\section{Result}

Among the 266 studied FNAC cases with cervical lymphadenopathy, $101(38 \%)$ cases were females and $165(62 \%)$ cases were males with male: female ratio of about 1:1.6 (Chart 1). In lymph node of cervical region Level-5 i.e. from posterior triangle involved most frequently (Table-2). The age wise distribution is given in (Table-3) in which high occurrence of reactive lymphadenitis in first decade, chronic granulomatous inflammation in third decade and malignancy in sixth decade noted. The cytological diagnoses were found to be benign in $206(76.7 \%$ ) cases and malignant in $62(23.3 \%)$ cases (Table-3). Among the benign cases occurrence of reactive lymphadenitis $67(25 \%)$, chronic granulomatous lymphadenitis $59(22 \%)$, acute suppurative inflammation 37 (14\%), tuberculous lymphadenitis $27(10 \%)$ and chronic non-specific in $14(5 \%)$ of cases noted. In malignant condition there is dominancy of metastatic squamous cell carcinoma found $23(8.6 \%)$ cases followed by metastatic adenocarcinoma 9 (3.4\%), primary non-hodgkins lymphoma $8(3 \%)$ and metastatic lesion from unknown malignancy were $8(3 \%)$ of cases and few miscellaneous cases given in (Table 3 ). The cytopathological results were then compared with the histopathological diagnoses of the corresponding excised lymph nodes. 25 cases of malignant lymphadenopathy diagnosed by cytology were consistent with histopathology.

\section{Discussion}

The lymphatic system is the important defense mechanism against the invading pathogens in the body through which diseases may not only be cleared away, but in case of malignant condition it plays conspicuous role 
Chart-1: Sex distribution of cervical lymphadenopathy cases.

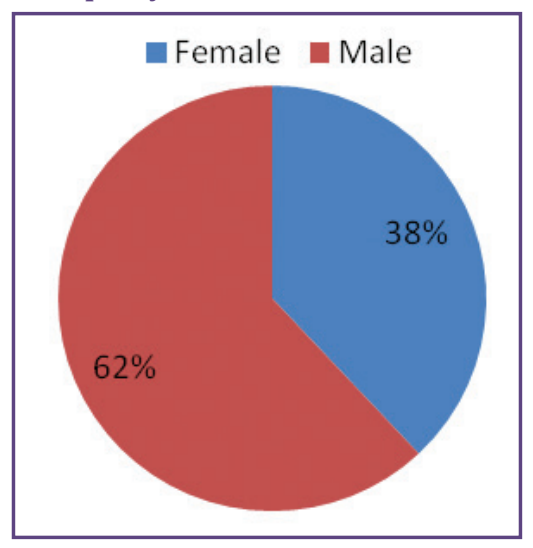

Chart-2: Level (Topographic distribution) of lymph nodes aspirated

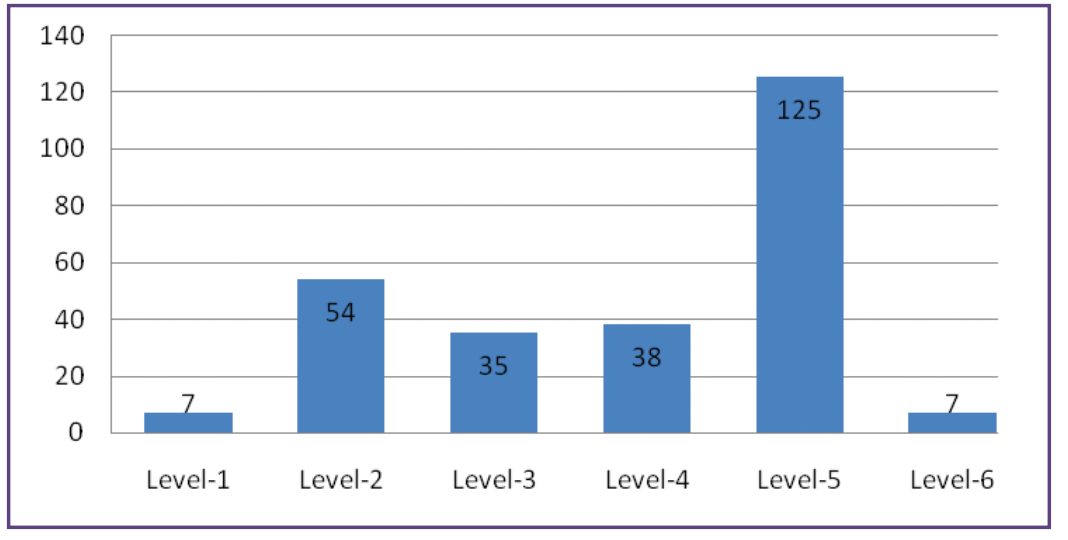

Table-3: Cytological diagnosis of cervical lymphadenopathy with age distribution.

\begin{tabular}{|c|c|c|c|c|c|c|c|c|c|c|}
\hline $\begin{array}{ll} & \text { Age (years) } \\
\text { Diagnosis } & \end{array}$ & $1-10$ & $11-20$ & $21-30$ & $31-40$ & $41-50$ & $51-60$ & $61-70$ & $71-80$ & $81-90$ & \\
\hline Reactive lymphadenopathy & 27 & 17 & 15 & 5 & 3 & - & - & - & - & 67 \\
\hline Granulomatous lymphadenopathy & 5 & 9 & 27 & 8 & 3 & 6 & - & - & 1 & 59 \\
\hline Acute suppurative lymphadenopathy & 7 & 4 & 8 & 7 & 5 & 5 & - & 1 & - & 37 \\
\hline Tuberculous lymphadenopathy & 1 & 9 & 6 & 7 & 2 & 2 & - & - & - & 27 \\
\hline Metastatic squamous cell carcinoma & - & - & 1 & 2 & 2 & 12 & 1 & 3 & 2 & 23 \\
\hline Chronic Non-specific lymphadenopathy & 5 & 4 & 3 & 2 & - & - & - & - & - & 14 \\
\hline Metastatic adenocarcinoma & - & - & - & 2 & 1 & 2 & 4 & - & - & 9 \\
\hline Primary lymphoma & 1 & 1 & - & 2 & 3 & - & 1 & - & - & 8 \\
\hline Metastatic unknown origin & 1 & - & 1 & 1 & - & 2 & 3 & - & - & 8 \\
\hline Metastaic neuroendocrine ca. & - & - & - & - & - & 1 & 2 & 1 & 1 & 5 \\
\hline Metastatic ducatal ca. breast & - & - & - & - & 1 & 1 & - & - & - & 2 \\
\hline Dermatopathic lymphadenopathy & - & - & 1 & - & - & - & - & - & - & 1 \\
\hline Kawasaki's lymphadenopathy & 1 & - & - & - & - & - & - & - & - & 1 \\
\hline Metastatic epithelial malignancy & - & - & - & - & 1 & - & - & - & - & 1 \\
\hline Metastatic malignant melanoma & - & - & - & - & 1 & - & - & - & - & 1 \\
\hline Metastatic nasopharyngeal ca. & - & 1 & - & - & - & - & - & - & - & 1 \\
\hline Metastatic thyroid malignancy & - & - & - & - & - & - & - & - & 1 & 1 \\
\hline Rosai-dorfman sinus histiocytosis & 1 & - & - & - & - & - & - & - & - & 1 \\
\hline Total & 49 & 45 & 62 & 36 & 22 & 31 & 11 & 5 & 5 & 266 \\
\hline
\end{tabular}




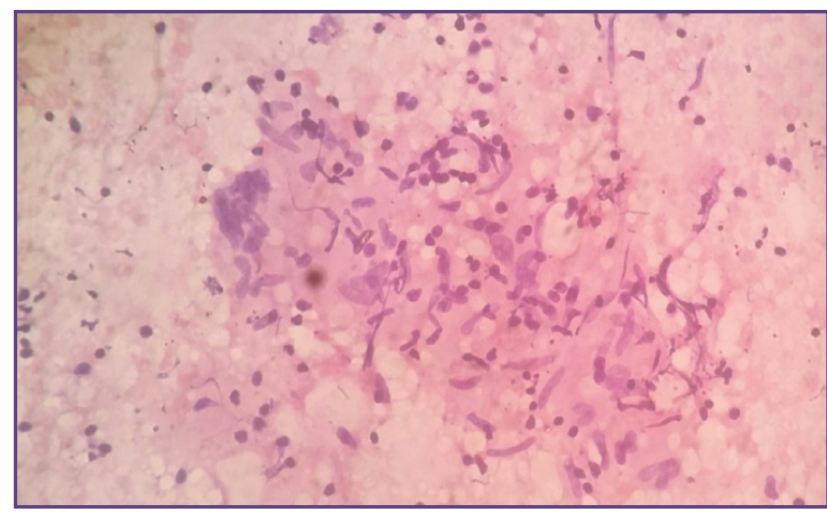

Fig. $1 \mathrm{H} \& \mathrm{E}$ stained smear in high power field showing granuloma formation.

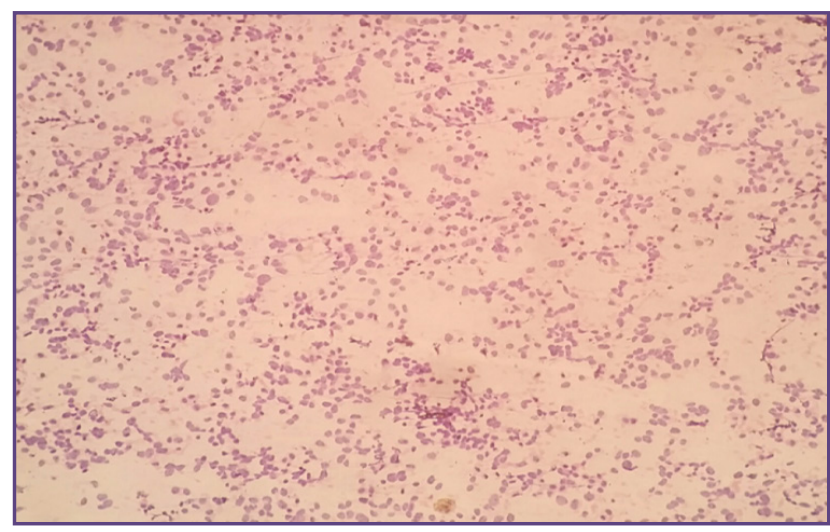

Fig. 2 H\&E stained smear in low power view showing (diffuse large B-cells) Non-Hodgkins lymphoma.

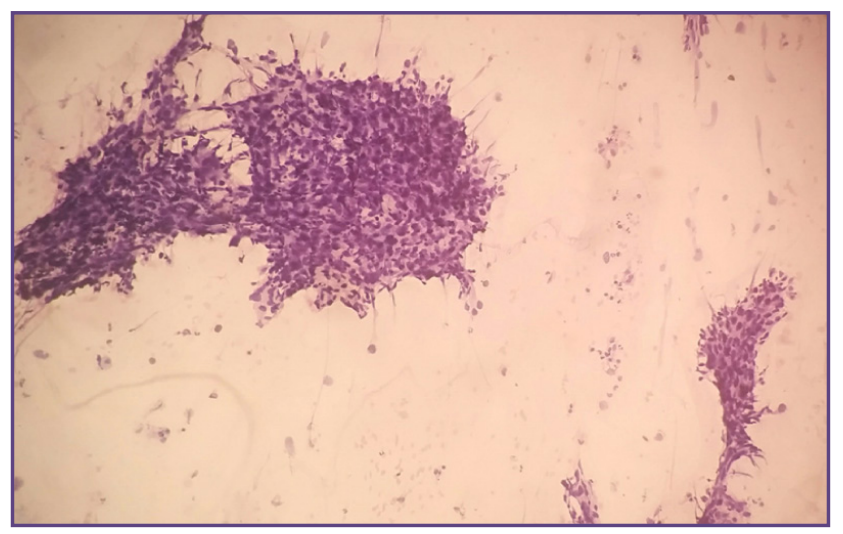

Fig. 3 H\&E stained smear in low power view showing sheets of malignant squamous epithelial cells in metastatic squamous cell carcinoma.

in spreading to the remote organs in the body. Cervical lymphadenopathy is a commonly encountered clinical condition requiring early and reliable diagnosis so that a proper treatment protocol can be started as early as possible. FNAC is a safe, quick and cost effective method for rapid evaluation of lymphadenopathy, reducing need of surgical biopsy. In the present study we evaluate 266 FNAC cases of cervical lymphadenopathy over a period of one year. The pattern of lesions varied from non-neoplastic lesions like tuberculous lymphadenopathy, reactive lymphadenopathy, acute suppurative lymphadenopathy, granulomatous lymphadenopathy, sinus histiocytosis and non-specific lymphadenopathy, to neoplastic lesions like metastatic lymphadenopathy and Lymphomas.

In our study, reactive lymphadenopathy was observed to be the most frequent diagnosis with 67/266 (25\%) cases. It was also the most frequent diagnosis in other studies and its incidence has been seen to range from $18.9 \%$ to $42 \%$. ${ }^{[18}$. ${ }^{19,20]}$ The second most frequent diagnosis in this study was observed to be chronic granulomatous inflammation with $59 / 266$ (22\%) cases (fig. 1). The incidence of granulomatous inflammation was observed to vary from $9.2 \%$ to $25.45 \%$ in other studies. ${ }^{[3,20]}$ Tuberculous lymphadenopathy diagnosed by cytology alone occurred in 27/266 (10\%) of our cases. Acute suppurative lymphadenopathy was observed to be a frequent occurrence with 37/266 (14\%) cases in our study. A study on tuberculous lymphadenitis done by Sarwar et al, ${ }^{[21]}$ found only necrosis without epithelioid granuloma in $35 \%$ cases. Surase et $\mathrm{a}^{\left[{ }^{[7]}\right.}$ in their study also found one of the cytological patterns of tuberculous lymphadenopathy to be only of necrotic material without epithelioid cells, or giant cells. In such cases Zeihl- Nielson's stain should be done to demonstrate Acid Fast Bacilli. Squamous Cell Carcinoma is known to be associated with necrosis. Finding only necrotic material on cytology without the evidence of malignant cells was noted in $2 \%$ cases, in a study conducted by Kiran Alam et al ${ }^{[22]}$ and $4.1 \%$ cases of the study by Bhagwan et al ${ }^{[23]}$. The present study also comprised 14/266 (5\%) cases diagnosed as non specific lymphadenopathy on cytology. This correlated somewhat with the study of Haque and Talukdar ${ }^{[9]}$ who found the incidence to be $2.63 \%$ of all lymph nodes aspirated. We diagnosed 59/266 (23\%) neoplastic lesions in the lymph nodes by FNAC, of which more cases of metastatic involvement 51/59 (86.6\%) rather than lymphoma 8/59 (13.3\%) were seen (fig.2). This was similar to other Indian studies. ${ }^{[19,22]}$ Other studies have found the incidence of neoplastic involvement to vary from $10.1 \%$ to $47.8 \%{ }^{[3,24]}$. A Brazilian FNAC study on lymph nodes diagnosed $79.4 \%$ metastasis and $14.2 \%$ lymphomas. ${ }^{[25]} \mathrm{A}$ study conducted in Egypt has reported more involvement by lymphomas $(80.3 \%)$ rather than metastatic diseases $(19.7 \%){ }^{[1]}$

Histopathological correlation (25/59 cases) was $100 \%$ in all our cases of metastatic malignancies of lymph nodes. Since most of our patients initially diagnosed cases of lymphoma belonged to the lower socio economic group, they referred to higher centre for immunohistochemistry 
when the patient could afford it. Most of the cases with the primary lymphoma either transferred to higher cancer institute or treatment defaulter. In our study the cervical group was the most common to be involved by metastasis and the primary was most often from the oral cavity, which was similar to other studies, ${ }^{[3,26]}$ with squamous cell carcinoma (fig.3) being the most common histological type. ${ }^{[2,22,23]}$ Rates for oral cavity, pharynx, oesophagus and male larynx are highest in India, probably due to the use of multiple tobacco products. Metastases from the lung, breast malignancy often due to lack of awareness about the malignancy and poor treatment compliance due to lower socioeconomic condition and illiteracy with social stigma ${ }^{[27]}$. Although we had a very low number of 25/266 cases (9.4\%) with histopathological correlation, we could diagnose reactive lymphadenopathy, sinus histiocytosis, chronic granulomatous lesion suggestive of tuberculosis and malignant lesions with high accuracy. The low number of biopsies was probably due to the clinician's satisfaction with the cytological diagnosis and correlates with other non- invasive diagnostic ailments like radiological and microbiological investigations. Thus FNAC of the lymph nodes can be used as an effective and simple diagnostic tool for cervical lymph node lesions.

\section{Conclusion}

FNAC is an important primary diagnostic tool for benign and malignant cervical lymphadenopathy. It is a safe, simple and inexpensive definite diagnostic procedure to render a prompt diagnosis, especially in lymph node aspirates, where biopsies are not done commonly. The limitations are with necrotic lymphadenopathy, heterogeneous swelling with limited representative aspirates, where if the clinician is unsatisfied with the cytological diagnosis, further workup like biopsy is required in order to make a reliable diagnosis. FNA evaluation in patient with no previously diagnosed malignancy should be interpreted conveniently and guide towards the proper workup and management of the primary lesion.

\section{Reference}

1. h VE, Harsharan SK, Jerald GZ. Fine needle aspiration biopsy of lymph nodes in the modern era: reactive lymphadenopathies. Pathol Case Rev 2007; 12(1):27-35

2. Das DK. Value and limitation of fine-needle aspiration cytology in diagnosis and classification of lymphomas: a review. Diagn Cytopathol 1999;21:240-9

3. Howlett DC, Harper B, Quante M, Berresford A, Morley M, Grant J. Diagnostic adequacy and accuracy of fine needle aspiration cytology in neck lump assessment: results from a regional cancer network over a one year period. J Laryngol Otol 2007;121(6):571-9
4. Kollur SM, El Hag IB. Fine needle aspiration cytology of metastatic nasopharyngeal carcinoma in cervical lymph nodes: comparison with metastatic squamous cell carcinoma and Hodgkin' and Non-Hodgkin' lymphoma. Diagn Cytopathol 2003;28:18-22

5. Al-Mulhim AS, Hafez NH, Tahoun NS, Reliability of fine needle aspiration cytology (FNAC)as a diagnostic tool in cases of cervical lymphadenopathy, Journal of the Egyptian National Cancer Institute 2011; 23(3), 105-114

6. Mohanty R, Wilkinson A, Utility of Fine Needle Aspiration Cytology of Lymph nodes IOSR J of Dental and Med Sciences (IOSR-JDMS) 2013;8(1):13-18

7. Hirachand S, Lakhey M, Akhter J, Thapa B. Evaluation of fine needle aspiration cytology of lymph nodes in Kathmandu Medical College, Teaching hospital. Kathmandu Univ Med J 2009; (26):139-42

8. Keit Al-Ghamdi AM, Al-Marzooq HM, Mohammad HA, Gharib IA. The role of fine needle aspiration cyto and imprint cytology in cervical lymphadenopathy.Saudi Med J 2004;25:862-5.

9. Haque MA, Talukder SI. Evaluation of fine needle aspiration cytology of lymph node in Mymensingh. Mymensingh Med J 2003;12(1):33-5

10. Raghuveer CV, Leekha IL, Pai MR, Adhikari P. Fine needle aspiration cytology versus fine needle sampling without aspiration. A prospective study of 200 cases. Indian J Med Sci 2002;56:431-9.

11. Shakya G, Malla S, Shakya KN, Shrestha R. A study of fine needle aspiration cytology of cervical lymph nodes. J Nepal Health Res Counc 2009;7(14):1-5.

12. Jeffers MD, Milton J, Herriot R, McKean M. Fine needle aspiration cytology in the investigation on Non Hodgkin' lymphoma. J Clin Pathol 1998;3:189-96.

13. Troxell ML, Charles DB, Athena MC, Yasodha N. Cytologic diagnosis of Burkitt lymphoma: role of ancillary techniques. Cancer Cytopathol 2005;105:310-8.

14. Sun HB, Zheng XF, Zhang J. Diagnostic accuracy of fine needle aspiration biopsy of cervical lymph node: a study of 580 cases. Zhonghua Bing Li Xue Za Zhi 2008;37(10):693-7.

15. Narang R, Pradhan S, Singh R. Place of fine needle aspiration cytology in the diagnosis of lymphadenopathy. Ind J Tuberc 1990;37(1):29-31.

16. Chieng DC, Cangiariella JF, Cohen JM. Fine needle aspiration cytology of Hodgkin disease: a study of 89 cases with emphasis on false negative cases. Cancer 2001;93(1):52-9.

17. Wakely PE. Fine needle aspiration cytopathology of malignant lymphoma. Clin Lab Med 1998; 18(3):541-7

18. Kochhar K, Patel B, Shah M. Pattern of Lymphadenopathy on Fine Needle Aspiration Cytology of Superficial Lymph Nodes (A Study of 150 Cases). JARBS 2012; 4: 288-292.

19. Khajuria R, Goswami KC, Singh K, Dubey VK. Pattern of Lymphadenopathy on Fine Needle Aspiration cytology 
in Jammu. JK Science Journal of Medical Education and Research 2006; 8:157-159.

20. Adhikari P, Sinha BK, Baskota DK. Comparison of fine needle aspiration cytology and histopathology in diagnosing cervical lymphadenopathies. AMJ 2011; 4: 97-99.

21. Sarwar A, Haque A, Aftab S, Mustafa M, Moatasim A, Siddique $\mathrm{S}$ et al. Spectrum of Morphological Changes in Tuberculous Lymphadenitis. International Journal of Pathology 2004; 2:85-89.

22. Alam K, Khan AH, Siddiqui FA, Jain A, Haider N. Fine Needle Aspiration Cytology (FNAC), a handy tool for metastatic lymphadenopathy. The Internet Journal of Pathology 2010;10. Available from: http://www.ispub.com/ journal/the_internet_journal_of_pathology

23. Bhagwan IN, Kane SV, Chinoy RF. Cytologic Evaluation of the Enlarged Neck Node: FNAC Utility in Metastatic
Neck Disease. The Internet Journal of Pathology 2007; 6:2. Available from: http://www.ispub.com/journal/the_internet journal_of_pathology

24. Ghartimagar D, Ghosh A, Ranabhat S, Shrestha MK, Narasimhan R, Talwar OP. Utility of fine needle aspiration cytology in metastatic lymph nodes. Journal of Pathology of Nepal 2011; 1: 92-95.

25. Martin MR, Santos GC. Fine Needle Aspiration Cytology in the diagnosis of superficial lymphadenopathy: a 5 year Brazilian experience. Diagn Cytopathol 2006; 34:130-134

26. Hoftr S, Muhle C, Brenner W, Sprenger E, Maune S. FineNeedle Aspiration Cytology of the Sentinel Lymph Node in Head and Neck Cancer. J Nucl Med 2002; 43:1585-9

27. Loya AC, Pragya AK, Arora A, Sundaram UC, Rao IS, Uppin SG et al. Lymph node metastasis of soft tissue tumours: A Cytomorphologic study. Acta Cytol 2007; 51: 153-160

*Corresponding author:

Dr. Alpeshpuri P. Goswami (M.D. Pathology), Associate professor in Department of Pathology,

Government Medical College, Bhavnagar, behind ST bus stand, Bhavnagar, 364001 INDIA

contact no.:9428125028

email: dralpeshgosai79@gmail.com

Date of Submission : 29.05.2017

Date of Acceptance : 30.10.2017

Financial or other Competing Interests: None.

Date of Publication : 27.01.2018 